INTERNATIONAL JOURNAL OF MULTIDISCIPLINARY RESEARCH AND ANALYSIS

ISSN(print): 2643-9840, ISSN(online): 2643-9875

Volume 04 Issue 11 November 2021

DOI: 10.47191/ijmra/v4-i11-30, Impact Factor: 6.072

Page No.- 1678-1681

\title{
Electrophysical Properties of Two Structured Polycrystal Silicon
}

\author{
Lutfiddin Omanovich Olimov ${ }^{1}$, Iqboljon Ibroximovich Anarboyev ${ }^{2}$ \\ ${ }^{1}$ Professor , Andijan Machine Building institute №56 Bobur Shox Ave, Andijan, Uzbekistan, 170100 \\ ORCID: 0000-0002-0135-5861 \\ ${ }^{2}$ PhD student, Andijan Machine-Building Institute №56 Bobur Shox Ave, Andijan, Uzbekistan, 170100
}

\begin{abstract}
The article first describes the results obtained in the study of the electrophysical and charge transfer processes of two structural polycrystalline silicon obtained by bonding silicon particles with sunlight. The results of the study show that the charge transfer processes in such structures are found to be different from each other. In particular, at T 300-800 K, a decrease in the surface area of both structures $\mu$ was observed, and the temperature dependence of $\rho$ and $\mathrm{n}$ differed from each other. For example, (a) in the surface area $\mathrm{T} \sim 300-350 \mathrm{~K}$ and $\mathrm{T} \sim 600-710 \mathrm{~K} \rho$ increases and $\mathrm{n}$ decreases, at $\mathrm{T} \sim 350-550 \mathrm{~K} \rho$ decreases and $n$ increases. Conversely, the surface area of the sample (b) is characterized by an increase in $\rho$ and a decrease in $n$ at $\mathrm{T} \leq 575 \mathrm{~K}$, a decrease in $\rho$ in the later stages of temperature increase, and an increase in $n$.
\end{abstract}

KEYWORDS: polycrystalline silicon, intergranular boundary, temperature, charge transfer processes, recombination centers.

\section{INTRODUCTION}

It is known that the physical properties of polycrystalline silicon (PC) based semiconductor devices or solar cells under certain conditions are explained by its microstructure and intergranular boundary (DCh) areas (e.g. [1-10], see also references given there). Studies have shown the potential for the production of relatively inexpensive and radiation-resistant solar cells or semiconductor devices as well as thermoelectric materials by controlling PC dimensional defects and introductory conditions in the DC field and charge transfer processes [1-7]. For example, it has been found that the introduction of additional alkali metal atoms in the DC field changes the conductivity [4,5], improves the conductivity, adsorption or dissociation of the input atoms eliminates the recombination centers in the $n$-layer and improves the diode characteristics of the $p-n$ structure $[6,7]$. These processes depend on the granule size or DCh field microstructure and are important in obtaining $p-n$ structures based on PK. For example, in the formation of the $p-n$ structure, the diffusion of phosphorus across the DCh domains is stronger than the granulation in the formation of $n$ layers by the introduction of phosphorus on the surface of $r$-type PK $[4,6,7]$. The process depends on the diffusion coefficient of the input atoms across the DCh areas relative to the granularity. Relatively speaking, the microstructure of DCh domains in PC size is formed in a similar way. However, in addition, for example, as noted above, it is of interest to study the charge transfer processes in two structural PCs that differ from the axial portion of the surface microstructure in which phosphorus is introduced during $\mathrm{n}$-layer formation. This method can control the diffusion of phosphorus across relatively DCh areas. This paper discusses the results obtained in the study of the electrophysical properties of two structural PCs obtained on the basis of powder technology.

\section{RESEARCH METHOD}

It is known that powder technology is one of the promising methods in obtaining polycrystalline semiconductor devices and thermoelectric materials due to its simplicity and the fact that it does not require complex technologies $[4,5-9,11]$. The novelty of the study is that it is the first study of the electrophysical properties and charge transfer processes of two structural PCs obtained by heating and bonding silicon particles prepared on the basis of powder technology with sunlight.

The electrophysical properties of PC were improved using traditional four-probe and modernized experimental devices and methods of Vander-Pau methods. The temperature dependence of specific resistance $(\rho)$, charge carrier mobility $(\mu))$, and concentration $(\mathrm{n}$ ) was studied in the process of temperature increase and decrease in the range $\sim 300 \div 800 \mathrm{~K}$. 


\section{Electrophysical Properties of Two Structured Polycrystal Silicon}

\section{ELECTROPHYSICAL PROPERTIES OF SAMPLES}

Figures 1 show the samples $\rho$ and and Figure 2 shows the temperature dependence of $n$ and $\mu$. In order to facilitate the interpretation of the results of the study, we introduce the designations of the surface area (a) and the surface area (b) where the sunlight falls on the sample. It should be noted that T 300 K of the sample (a) surface area $\rho \sim 191,9 \mathrm{Om} \cdot \mathrm{sm}, n \sim 3,11 \cdot 10^{18} \mathrm{sm}^{3}$, $\mu \sim 0,0245 \mathrm{sm}^{2} / V s ;(b)$, surface area $\rho \sim 452 \mathrm{Om} \cdot \mathrm{sm}, n \sim 5,52 \cdot 10^{17} \mathrm{sm}^{3}, \mu \sim 0,025 \mathrm{sm}^{2} / V s$ accepts values. The results of the study show that a decrease in $\mu$ (line 3 ) in both (a) and (b) surface area was detected. However, in the surface area (a) and (b) the temperature dependence of $\rho$ and $\mathrm{n}$ differs from each other. For example, if (a) increases in the surface area at $\sim 300 \div 350 \mathrm{~K}, \mathrm{n}$ decreases (case a-b), if $\rho$ decreases at $\mathrm{T} \sim 350 \div 550 \mathrm{~K}, \mathrm{n}$ increases (case b-c). Then, in the $50 \mathrm{~K}$ range, both parameters change relatively steadily (line 1). If $\rho$ increases again at $-600 \div 710 \mathrm{~K}$, it decreases to $n 775 \mathrm{~K}$ (c-d condition). At $T \sim 710 \div 775 \mathrm{~K}$, the sag change of $\rho$ (d-e state) was detected. Conversely, if the surface area of the sample (b) increases at $\rho \mathrm{T} \leq 575 \mathrm{~K}$ (a-b 'state), $\mathrm{n}$ decreases (a-b-s' state), in the later stages of temperature increase (b-state) As $\rho$ decreases, $n$ (state c'-d ') increases (line 2). The results of the study can be interpreted as follows based on the microstructure model and zoning diagram of the sample shown in Figure 3. Figure 3 shows a model of the microstructure structure of the surface areas (a) and (b) of the sample and a zone diagram of the DC area (c). The structure of the sample can be conditionally determined by its granularity (1) and DC areas (2). The process of charge transfer in a polycrystalline semiconductor is mainly explained by the DCh field [1, 2]. The charge carriers move from area A to area B through the DCh fields (2) (Figure 3). In the WC field, strongly unbound and disconnected bonds form recombination centers of different Eip energy levels (Fig. 3c). They are localized in the DCs and create a barrier effect.

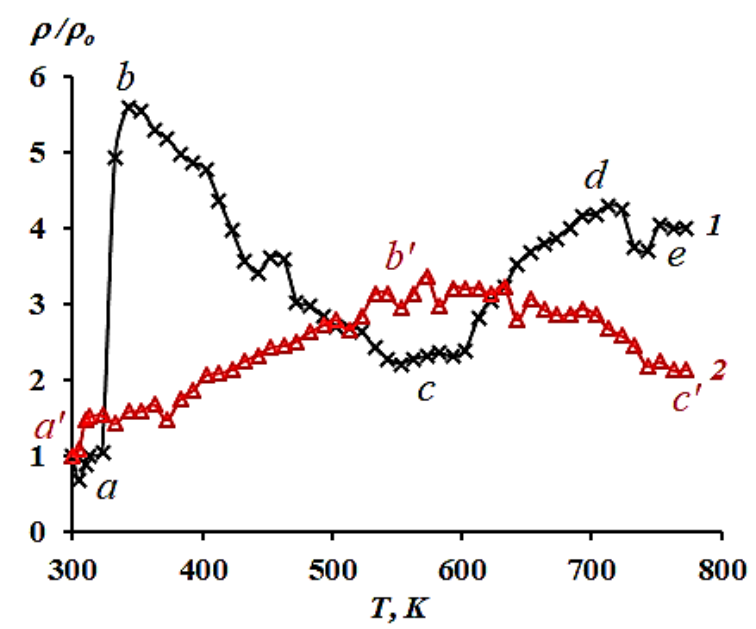

Figure 1. Temperature dependence of specific resistance.

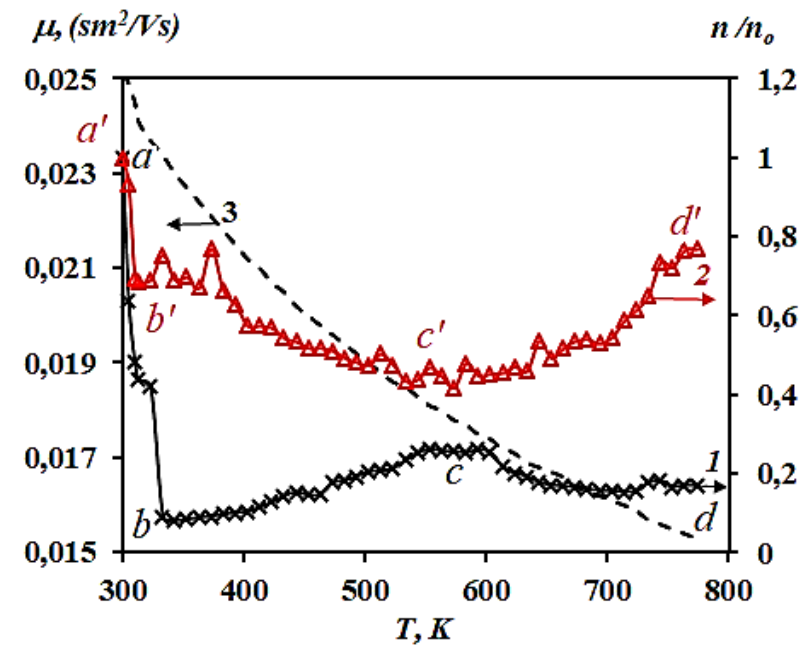

Figure 2. Temperature dependence of the concentration and mobility of charge carriers.

The capture and re-release of charge carriers in localized traps results in a change in the potential barrier height $(\varphi)$, which in turn $\rho$. According to the Setto model, the following expression can be written for $\rho[1,2]$ : 


$$
\rho=\frac{k}{q\langle a\rangle A^{*} T} \exp (q \varphi / k T)
$$

where, $q$ - electron charge, $k$-Boltsman's constant, $\langle a\rangle$ - granularity measure, $A^{*}$ - Richardson's efficiency constant, - temperature.

Depending on the amount of charge $\left(Q_{i}\right)$ trapped in $\varphi$ gi localized traps in the WC fields:

$$
\varphi=\frac{Q_{i}^{2}}{8 \varepsilon \varepsilon_{0} q N_{G}}
$$

where $N_{G}$ is the concentration of electrically active alloyed inputs, $\varepsilon$ and $\varepsilon_{0}$ are the relative dielectric constant of the medium and the vacuum, respectively.
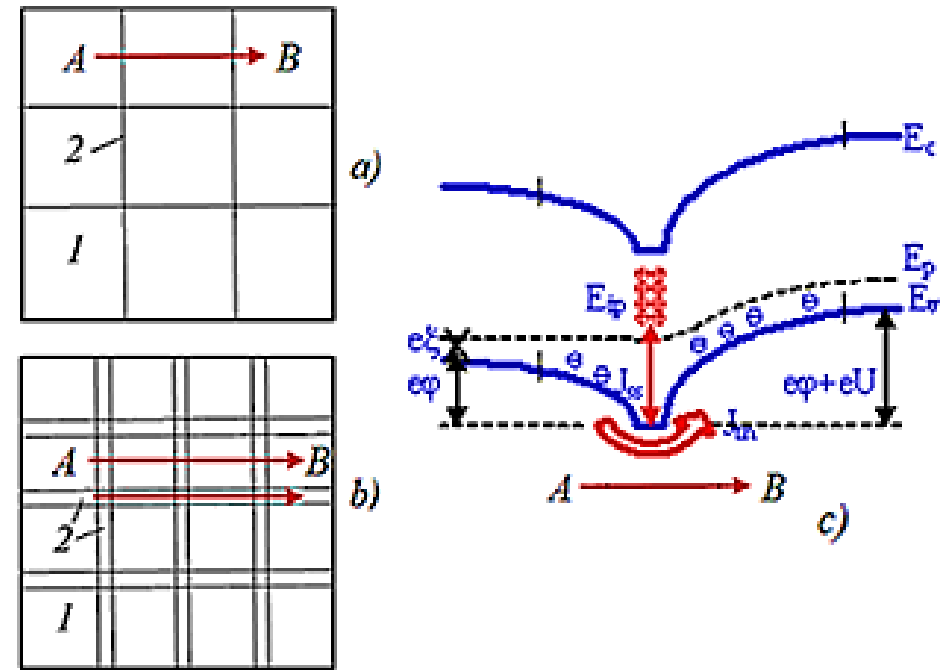

Figure 3. The microstructure model of the sample and the mechanism for explaining the charge transfer process.

Expression (2) shows that an increase in $Q_{i}$ in the DC field (field 2) leads to an increase in $\varphi$, which in turn leads to an

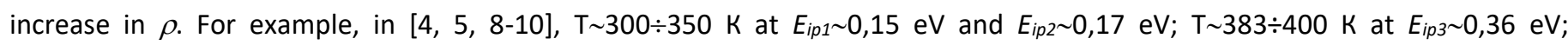
$\mathrm{T} \sim 400 \div 680 \mathrm{~K}$ at $E_{i p 3} \sim 0,3 \mathrm{eV}$ it was found that the appearance of levels leads to an increase in PK $\rho$. It has also been shown that the electrophysical and photoelectric properties of PK and the p-n structures derived from it depend on the concentration of localized traps at the $E_{i p}$ energy level. The comments made are also relevant to our results. However, in our case, it was observed that the change in $\rho$ differed according to the microstructure of the surface areas (a) and (b). In our opinion, this may be due to the nature of the manifestation of recombination centers in the WCs in the surface areas (a) and (b). First, (a) consider the surface area microstructure model and charge transfer processes. As mentioned above, (a) the surface area consists of a microstructure in which the silicon particles are solid and cohesive. In our opinion, in the initial stage of temperature increase, i.e. at T 300 $\div 350 \mathrm{~K}$, energy levels $\mathrm{E} \sim 0.15 \mathrm{eV}$ and $\mathrm{E} \sim 0.17 \mathrm{eV}$ appear. An increase in $\mathrm{Q}_{i}$ captured in them leads to a decrease in $n$ and an increase in $\rho$ (Figures 1 and 2, case $a-b$ ). As the temperature increases, the Eip energy level increases relative to the concentration of localized traps $n$ separated from the valence band. This leads to a decrease in $\rho$ at $\mathrm{T} \sim 350 \div 550 \mathrm{~K}$ (in area b-c). At T $550 \div 600 \mathrm{~K}$, the concentration of $\mathrm{n}$ and localized traps separated from the valence band is equal, and in this case $\rho$ changes steadily. In the later stages of the temperature increase, the concentration of localized traps of the $E_{i p}$ energy level in the DC fields again increases with respect to $\mathrm{n}$ separated from the valence band. $\mathrm{T}-600 \div 710 \mathrm{~K}$ leads to an increase in $Q_{i}$ and $\rho$ (case c-d). The appearance of new $E_{i p}$ energy-level recombination centers in accordance with the temperature may lead to a sag change of $\rho$ (d-e state) at $\mathrm{T} \sim 710 \div 775 \mathrm{~K}$.

Now (b) consider the charge transfer processes in the surface area. As mentioned above, (b) the surface area (a) consists of a rougher surface than the surface area. Its microstructure (a) differs relative to the surface area. That is, the width of the DC field (a) is wider than the surface area (Fig. 2a). In this case, the charge transfer process can be carried out simultaneously through the DC fields and across the DC fields [3-5, 9]. Due to the relatively wider DC field, the area will have a higher number of strongly unbound and disconnected bonds that form localized traps at the $E_{i p}$ energy level. Accordingly, there is also a high probability of the formation of localized traps of the $E_{i p}$ energy level at different temperatures. The results of the study show that the increase in $\rho$ at $\mathrm{T} \leq 575 \mathrm{~K}$ (a-b 'state) is consistent with the results obtained in the works [3-5, 9]. That is, at 


\section{Electrophysical Properties of Two Structured Polycrystal Silicon}

$\mathrm{T} \sim 300 \div 600 \mathrm{~K}$, recombination centers of $E_{i p 1} \sim 0,15 \mathrm{eV}, E_{i p 2} \sim 0,17 \mathrm{eV}, E_{i p 3} \sim 0,36, E_{i p 3} \sim 0,3 \mathrm{eV}$ appear in series. An increase in the amount of charge carriers $Q_{i}$ in them leads to an increase in $\rho$ at $\mathrm{T} \sim 600 \div 710 \mathrm{~K}$ (a-b 'state). A decrease in $n$ in the same temperature range ( $a$ '- $b^{\prime}-s^{\prime}$ state) and a jump in certain temperatures may confirm the observations. That is, the jump change at $\mathrm{T} \sim 325 \div 400 \mathrm{~K}$ corresponds to the formation of levels $E_{i p 1} \sim 0,15 \mathrm{eV}, E_{i p 2} \sim 0,17 \mathrm{eV}$ and $E_{i p 3} \sim 0,36 \mathrm{eV}$. In the later stages of temperature rise, localized traps of the $E_{i p}$ energy level in the $\mathrm{DC}$ field (2) begin to ionize. As a result, the charge carriers simultaneously move from area A to area B through the DC fields (2) and along the localized traps (Fig. 3b). The migration of charge carriers along localized traps leads to the formation of $J_{s s}$ (Fig. 3s) [3, 4, 9]. This current occurs during the capture and rerelease of localized traps in the $E_{i p}$ energy level above the $E_{f}$ farm level as the charge carriers move from left to right. $J_{s s}$ depends on the total conductivity $\left(\mathrm{Y}_{\mathrm{ss}}\right)$ of the current traps, i.e., the total conductivity and the potential barrier height $(\delta \varphi)$, which depends on the distribution of the traps across the granular surfaces and energy.

$$
J_{s S}=Y_{s S} \delta \varphi
$$

In this case, $\varphi$ does not decrease, $Y_{s s}$ increases. In studies $[3,4,9]$, it has been found that the migration of charge carriers along localized traps leads to an increase in $\mathrm{Y}_{\text {ss. }}$ In our opinion, the above comments are valid for $\rho$ (Fig. 1, b'-condition). An increase in the ionization process of localized traps leads to an increase in $n$ (Fig. 2, line 2, c'-d 'state).

\section{CONCLUSION}

In summary, samples from which silicon particles are attached to sunlight have two different structures of polycrystalline silicon, and the microstructure of the surfaces on which sunlight falls and does not fall, as well as the charge transfer processes, are different. The method of obtaining PC by attaching silicon particles with sunlight can play an important role in obtaining two structural semiconductor materials for microelectronics and photovoltaics.

\section{REFERENCES}

1) Harbek G. 1989 (eds) Polycrystalline semiconductors. Physical properties and applications: (Moscow, Russia).

2) L. Kazmerskii. 1983 (eds) Thin Polycrystalline and Amorphous Films. Physics and Applications. (Moscow, Russia).

3) Olimov L.O., Abdurakhmanov B.M. The Features of Impurity Thermal-Photovoltaic and thermal-Voltaic Effect of Polycrystalline Structures. Advances in Energy and Power USA. 1(2): 51-55, 2013.

4) Olimov L.O. 2016 (eds) "The grain boundaries of polycrystalline silicon: microwaves, charge states and p-n-junction". Autoreferaty of doctoral dissertation. (Uzbekistan)

5) Olimov L.O, Abdurakhmanov B.M, Teshaboev A. 2014 Influence of alkali metal atoms on transport of charge carriers in the between grain boundaries of polycrystalline silicon. J. Material Science, 114.

6) Olimov, L.O. 2012 Effect of alkali metals on the electronic properties of grain boundaries on a polycrystalline silicon surface. J. Semiconductors, 46898.

7) Olimov, L.O. 2010 Adsorption of alkali metals and their effect on electronic properties of grain boundaries in bulk of polycrystalline silicon. J. Semiconductors, 44602.

8) Abdurakhmanov, B.M., Olimov, L.O., Saidov, M.S. 2008 Electrophysical properties of solar polycrystalline silicon and its $\mathrm{n}^{+}$-p structures at elevated temperatures. J. Applied Solar Energy 4446.

9) Olimov, L.O. 2010 Model of the grain boundary in p-n structures based on polycrystalline semiconductors. J. Applied Solar Energy, 46118.

10) Saidov, M.S., Abdurakhmanov, B.M., Olimov, L.O. 2007 Impurity thermovoltaic effect in the grain boundaries of a polycrystalline silicon solar cell. J. Applied Solar Energy, 4203.

11) Olimov L.O., Anarboev I.I., Mamirov A., Omonboev F.L., Omonboeva M.L. 2021 (eds) Patent UZ № FAP 01593 "Method of preparation of thermoelectric material". 